\title{
APPEASEMENT RECONSIDERED: \\ INVESTIGATING THE MYTHOLOGY OF THE 1930s
}

Jeffrey Record

乙

August 2005 